Canad. Math. Bull. Vol. 42 (4), 1999 pp. 507-512

\title{
Index of volume 42
}

A 257 Austin, David, Dale Rolfsen Homotopy of Knots and the Alexander Polynomial

417 Aziz-Ul-Auzeem, Abdul, B. A. Zarger Some Properties of Rational Functions with Prescribed Poles

B 129 Baker, Andrew Hecke Operations and the Adams E $E_{2}$-Term Based on Elliptic Cohomology

3 Beauzamy, Bernard How the Roots of a Polynomial Vary with Its Coefficients: A Local Quantitative Result

427 Berndt, Bruce C., Heng Huat Chan Ramanujan and the Modular j-Invariant

441 Berrizbeitia, P., P. D. T. A. Elliott Product Bases for the Rationals

401 Blau, Philip S. See Gordon A. Swain

445 Bochnak, J., W. Kucharz Smooth Maps and Real Algebraic Morphisms

139 Bonet, José, Paweł Domański, Mikael Lindström Essential Norm and Weak Compactness of Composition Operators on Weighted Banach Spaces of Analytic Functions

149 Boyer, S., X. Zhang A Note on Finite Dehn Fillings

452 Bradley, Sean Finite Rank Operators in Certain Algebras

13 Brendle, Jörg Dow's Principle and Q-Sets

25 Brown, Tom C., Ronald L. Graham, Bruce M. Landman On the Set of Common Differences in van der Waerden's Theorem on Arithmetic Progressions

C 155 Campbell, H. E. A., A. V. Geramita, I. P. Hughes, R. J. Shank, D. L. Wehlau Non-Cohen-Macaulay Vector Invariants and a Noether Bound for a Gorenstein Ring of Invariants

427 Chan, Heng Huat See Bruce C. Berndt

263 Choie, Youngju, Min Ho Lee Mellin Transforms of Mixed Cusp Forms

37 Christensen, Ole Operators with Closed Range, Pseudo-Inverses, and Perturbation of Frames for a Subspace

162 Cobos, Fernando, Thomas Kühn Lorentz-Schatten Classes and Pointwise Domination of Matrices 
D 274 Dădărlat, Marius, Søren Eilers The Bockstein Map is Necessary

285 Deng, Peiming On Kloosterman Sums with Oscillating Coefficients

46 Dijkstra, Jan J. Generic Partial Two-Point Sets Are Extendable

169 Ding, Hongming Heat Kernels of Lorentz Cones

139 Domański, Pawet See José Bonet

E 52 Edmonds, Allan L. Embedding Coverings in Bundles

274 Eilers, Søren See Marius Dădărlat

56 Elbert, Árpád, Panayiotis D. Siafarikas On the Square of the First Zero of the Bessel Function $J_{\nu}(z)$

441 Elliott, P. D. T. A. See P. Berrizbeitia

F 174 Ferrero, Miguel, Alveri Sant'Ana Rings With Comparability

184 Fiorito, Giovanni On Arithmetic Means of Sequences Generated by a Periodic Function

G 155 Geramita, A. V. See H. E. A. Campbell

190 Gilmer, Patrick M. Topological Quantum Field Theory and Strong Shift Equivalence

68 Gittenberger, Bernhard, Jörg M. Thuswaldner The Moments of the Sum-Of-Digits Function in Number Fields

78 González, Josep Fermat Jacobians of Prime Degree over Finite Fields

25 Graham, Ronald L. See Tom C. Brown

291 Grubb, D. J., Tim LaBerge Spaces of Quasi-Measures

198 Guadalupe, José J., Mario Pérez, Juan L. Varona Commutators and Analytic Dependence of Fourier-Bessel Series on $(0, \infty)$

H 463 Hofmann, Steve, Xinwei Li, Dachun Yang A Generalized Characterization of Commutators of Parabolic Singular Integrals

155 Hughes, I. P. See H. E. A. Campbell

J 298 Jespers, Eric, Jan Okniński Semigroup Algebras and Maximal Orders 
K 307 Kapovich, Michael, John J. Millson On the Moduli Space of a Spherical Polygonal Linkage

321 Kikuchi, Masato Averaging Operators and Martingale Inequalities in Rearrangement Invariant Function Spaces

335 Kim, Goansu, C. T. Yang Cyclic Subgroup Separability of HNN-Extensions with Cyclic Associated Subgroups

87 Kittaneh, Fuad Some norm inequalities for operators

344 Koldobsky, Alexander Positive Definite Distributions and Subspaces of $L_{p}$ With Applications to Stable Processes

445 Kucharz, W. See J. Bochnak

162 Kühn, Thomas See Fernando Cobos

97 Kwon, E. G. On Analytic Functions of Bergman BMO in the Ball

L 291 LaBerge, Tim See D. J. Grubb

25 Landman, Bruce M. See Tom C. Brown

209 Lanteri, Antonio, Hidetoshi Maeda Ample Vector Bundles of Curve Genus One

263 Lee, Min Ho See Youngju Choie

463 Li, Xinwei See Steve Hofmann

139 Lindström, Mikael See José Bonet

M 209 Maeda, Hidetoshi See Antonio Lanteri

354 Marshall, Murray A. A Real Holomorphy Ring without the Schmüdgen Property

359 Martin, W. J., D. R. Stinson A Generalized Rao Bound for Ordered Orthogonal Arrays and $(t, m, s)$-Nets

371 Marubayashi, H., A. Ueda Prime and Primary Ideals in a Prüfer Order in a Simple Artinian Ring with Finite Dimension over its Center

307 Millson, John J. See Michael Kapovich

N 104 Nikolskaia, Ludmila Instabilité de vecteurs propres d'opérateurs linéaires

380 Nurmela, Kari J., Patric R. J. Östergård, Rainer aus dem Spring Asymptotic Behavior of Optimal Circle Packings in a Square

O 298 Okniński, Jan See Eric Jespers

380 Östergård, Patric R. J. See Kari J. Nurmela 
P 214 Paeng, Seong-Hun, Jong-Gug Yun Conjugate Radius and Sphere Theorem

221 Peide, Liu, Eero Saksman, Hans-Olav Tylli Boundedness of the q-Mean-Square Operator on Vector-Valued Analytic Martingales

198 Pérez, Mario See José J. Guadalupe

386 Polat, Norbert Minimal Separators

478 Pruss, Alexander R. A Remark On the Moser-Aubin Inequality For Axially Symmetric Functions On the Sphere

R 118 Rao, T.S. S. R. K. Points of Weak*-Norm Continuity in the Unit Ball of the Space $\mathrm{WC}(K, X)^{*}$

257 Rolfsen, Dale See David Austin

231 Rush, David E. Generating Ideals in Rings of Integer-Valued Polynomials

S 221 Saksman, Eero See Liu Peide

174 Sant'Ana, Alveri See Miguel Ferrero

393 Savin, Gordan A Class of Supercuspidal Representations of $G_{2}(k)$

486 Sawyer, $P$. Spherical Functions on $\mathrm{SO}_{0}(p, q) / \mathrm{SO}(p) \times \mathrm{SO}(q)$

155 Shank, R. J. See H. E. A. Campbell

56 Siafarikas, Panayiotis D. See Árpád Elbert

125 Smith, Larry Modular Vector Invariants of Cyclic Permutation Representations

380 Spring, Rainer aus dem See Kari J. Nurmela

359 Stinson, D. R. See W. J. Martin

401 Swain, Gordon A., Philip S. Blau Lie Derivations in Prime Rings With Involution

T 412 Tai, Yung-Sheng Peirce Domains

237 Thompson, A. C. On Benson's Definition of Area in Minkowski Space

68 Thuswaldner, Jörg M. See Bernhard Gittenberger

221 Tylli, Hans-Olav See Liu Peide

U 371 Ueda, A. See H. Marubayashi

V 198 Varona, Juan L. See José J. Guadalupe 
W 248 Weber, Christian The Classification of $\operatorname{Pin}_{4}$-Bundles over a 4-Complex 155 Wehlau, D. L. See H. E. A. Campbell

Y 335 Yang, C. T. See Goansu Kim 463 Yang, Dachun See Steve Hofmann 214 Yun, Jong-Gug See Seong-Hun Paeng

Z 499 Zaharia, Alexandru Characterizations of Simple Isolated Line Singularities 417 Zarger, B. A. See Abdul Aziz-Ul-Auzeem

149 Zhang, $X$. See S. Boyer 